\section{(2) OPEN ACCESS}

\title{
Clinical outcome of COVID-19 in patients with adult congenital heart disease
}

\author{
Markus Schwerzmann (D) ,' Francisco Javier Ruperti-Repilado (D) ,' \\ Helmut Baumgartner, ${ }^{2}$ Berto Bouma, ${ }^{3}$ Judith Bouchardy, ${ }^{4,5}$ Werner Budts, ${ }^{6,7}$ \\ Laurence Campens (D) , ${ }^{8}$ Massimo Chessa (D) , ${ }^{9}$ Maria Jesús del Cerro Marin, ${ }^{10}$ \\ Harald Gabriel, ${ }^{11}$ Pastora Gallego (1) , ${ }^{12}$ Rocio Garcia-Orta, ${ }^{13}$ Ana Elvira Gonzalez, ${ }^{14}$ \\ Annette Schophuus Jensen (D) , ${ }^{15}$ Magalie Ladouceur, ${ }^{16}$ Berta Miranda-Barrio, ${ }^{17}$ \\ Marielle Morissens, ${ }^{18}$ Agnes Pasquet, ${ }^{19}$ Joaquín Rueda, ${ }^{20}$ \\ Annemien E van den Bosch (D) , ${ }^{21}$ Heleen Berdina van der Zwaan, ${ }^{22}$ \\ Daniel Tobler (1) , ${ }^{23}$ Matthias Greutmann, ${ }^{24}$ on behalf of EPOCH
}

\begin{abstract}
- Additional material is published online only. To view, please visit the journal online (http://dx.doi.org/10.1136/ heartjnl-2020-318467).

For numbered affiliations see end of article.
\end{abstract}

\section{Correspondence to} Professor Markus Schwerzmann, Center for Congenital Heart Disease, Inselspital University Hospital, Bern 3010, Switzerland; markus.schwerzmann@med. unibe.ch

DT and MG are joint senior authors.

Received 15 October 2020 Revised 8 February 2021 Accepted 16 February 2021 Published Online First 8 March 2021

\section{Linked}

http://dx.doi.org/10.1136/ heartjnl-2021-319054

\section{Check for updates}

(C) Author(s) (or their employer(s)) 2021. Re-use permitted under CC BY-NC. No commercial re-use. See rights and permissions. Published by BMJ.

To cite: Schwerzmann M, Ruperti-Repilado FJ, Baumgartner $\mathrm{H}$, et al. Heart 2021;107:1226-1232.

\section{ABSTRACT}

Aims Patients with adult congenital heart disease (ACHD) are a potentially vulnerable patient cohort in case of COVID-19. Some cardiac defects may be associated with a poor COVID-19 outcome. Risk estimation in ACHD is currently based on expert opinion. The aim of this study was to collect clinical outcome data and to identify risk factors for a complicated course of COVID-19 in patients with ACHD.

Methods Twenty-five ACHD centres in nine European countries participated in the study. Consecutive patients with ACHD diagnosed with COVID-19 presenting to one of the participating centres between 27 March and 6 June 2020 were included. A complicated disease course was defined as hospitalisation for COVID-19 requiring non-invasive or invasive ventilation and/or inotropic support, or a fatal outcome.

Results of 105 patients with a mean age of $38 \pm 13$ years (58\% women), 13 had a complicated disease course, of whom 5 died. In univariable analysis, age (OR $1.3,95 \% \mathrm{Cl} 1.1$ to 1.7 , per 5 years), $\geq 2$ comorbidities (OR 7.1,95\% Cl 2.1 to 24.5), body mass index of $>25 \mathrm{~kg} / \mathrm{m}^{2}$ (OR $7.2,95 \% \mathrm{Cl} 1.9$ to 28.3 ) and cyanotic heart disease (OR $13.2,95 \% \mathrm{Cl} 2.5$ to 68.4) were associated with a complicated disease course. In a multivariable logistic regression model, cyanotic heart disease was the most important predictor (OR 60.0, $95 \% \mathrm{Cl} 7.6$ to 474.0 ).

Conclusions Among patients with ACHD, general risk factors (age, obesity and multiple comorbidities) are associated with an increased risk of complicated COVID-19 course. Congenital cardiac defects at particularly high risk were cyanotic lesions, including unrepaired cyanotic defects or Eisenmenger syndrome.

\section{INTRODUCTION}

The first wave of COVID-19 caused by SARS-CoV-2 hit Europe in March 2020. As of the end of September, more than 3.3 million cases with $>190$ 000 deaths have been counted by the European Centre for Disease Prevention and Control. ${ }^{1}$ As a response to the pandemic, European countries have developed strategies for minimising transmission of the virus, spread of the infection and disease-related morbidity and mortality. This includes the identification of vulnerable patients with underlying medical conditions associated with poor COVID-19 outcome, requiring particular protection.

Patients with adult with congenital heart disease (ACHD) represent such a potentially vulnerable patient cohort. With an estimated ACHD prevalence of 3000 per million adults, more than 2.5 million adults with congenital cardiac defects are currently living in Europe. ${ }^{2}$ A cure of the congenital cardiac defect by surgery or other interventions is still exceptional and many patients with ACHD face a lifelong increased risk of cardiovascular complications, such as heart failure, arrhythmias, pulmonary hypertension and premature death. ${ }^{3}{ }^{4}$ Respiratory diseases-in particular, pneumonia-are the most common non-cardiac cause of death in patients with ACHD, especially among patients with genetic disorders. ${ }^{5}$ Therefore, yearly influenza vaccination is recommended for most patients with ACHD. ${ }^{6}$

Patients with ACHD with simple lesions and no genetic disorder may not be at higher COVID-19 risk than the general population, whereas patients with more complex disease (eg, Fontan physiology, cyanotic heart disease, defects with impaired subpulmonary or subaortic ventricular function) may be at risk of haemodynamic compromise, hypoxia or paradoxical embolism if COVID-19. related complications occur. These uncertainties have led to substantial concerns among patients, relatives and treating physicians. In the absence of reported data, risk estimation is currently based on expert opinion. ${ }^{7}$ The aims of this study were to collect clinical outcome data and to identify risk factors for a complicated course of COVID-19 in patients with ACHD in European reference centres.

\section{METHODS}

As part of a research initiative within the European Collaboration for Prospective Outcome Research in Congenital Heart Disease (EPOCH, https:// 
www.sacher-registry.com/epoch/), this collaborative study was launched among different ACHD centres across Europe.

A total of 25 ACHD centres in nine European countries participated in the study. All patients with ACHD diagnosed with COVID-19 (positive test for SARS-CoV-2 by means of PCR test, antibody and SARS-CoV-2 antigen-based ELISA) or strong clinical suspicion (based on symptoms and thoracic CT findings) presenting to or contacting one of the participating centres were included. The study started on March 27 and data reported until 6 June 2020 were analysed. Only patients actively reporting to their centres or hospitalised for COVID-19 at the participating centres were included.

The following data were collected: type of cardiac defect, complexity of cardiac defect according to the most recent European ACHD guidelines, ${ }^{8}$ defect-related residual cardiac problems, gender, age (in years), weight category, most clinically relevant comorbidity according to the treating cardiologist's perception, number of comorbidities, clinical course and outcome. Definitions for categories within these different study characteristics are provided as online supplemental material 1. A complicated disease course was defined as hospitalisation for COVID-19 requiring non-invasive or invasive ventilation and/or inotropic support, extracorporeal membrane oxygenation or a fatal outcome. In patients with a complicated disease course, further detailed information on patient characteristics and disease course was obtained from the treating physicians of the pertinent centre. The presumed causal relationship between the congenital heart defect and the COVID-19 outcome was adjudicated after discussion with the treating physician and within the steering committee of the study (MS, JR, DT and MG). The following categories were discriminated: death or complicated disease course due to SARS-CoV-2 infection (COVID-19 was the main reason for outcome), death or complicated disease course with coincidental SARS-CoV-2 infection (pre-existing disease was the main reason for outcome).

Table 1 Characteristics of patients with ACHD and COVID-19 disease course

\begin{tabular}{|c|c|c|c|c|}
\hline & Overall $(\mathrm{N}=105)$ & Uncomplicated course $(n=92)$ & Complicated course $(n=13)$ & $P$ value* \\
\hline Age (years) & $38 \pm 13$ & $37 \pm 12$ & $47 \pm 13$ & 0.009 \\
\hline Age range (years) & $16-75$ & $16-75$ & $21-64$ & \\
\hline Female gender, $\mathrm{n}(\%)$ & $61(58)$ & $56(61)$ & $5(38)$ & 0.125 \\
\hline BMI $\left(\mathrm{kg} / \mathrm{m}^{2}\right), \mathrm{n}(\%)$ & & & & 0.001 \\
\hline $\mathrm{BMI}<25$ & $66(63)$ & $63(68)$ & $3(23)$ & \\
\hline BMI 25-30 & $26(25)$ & $21(23)$ & $5(38)$ & \\
\hline $\mathrm{BMI}>30$ & $13(12)$ & $8(9)$ & $5(38)$ & \\
\hline Comorbidities, n (\%) & & & & 0.003 \\
\hline None & $62(59)$ & $58(63)$ & $4(31)$ & \\
\hline One & $23(22)$ & $21(23)$ & $2(15)$ & \\
\hline Two or three & $20(19)$ & $13(14)$ & $7(54)$ & \\
\hline \multicolumn{5}{|l|}{ Cardiac defect } \\
\hline Cyanotic heart disease or ES & 7 & 3 & 4 ( 2 deaths) & \\
\hline Fontan circulation & 5 & 4 & 1 & \\
\hline TGA & 9 & 8 & 1 & \\
\hline Other complex defect & 4 & 4 & 0 & \\
\hline Tetralogy of Fallot & 18 & 17 & 1 (1 death) & \\
\hline Ebstein anomaly & 4 & 4 & 0 & \\
\hline Aortic coarctation & 10 & 9 & 1 & \\
\hline Other moderately complex defect & 8 & 7 & 1 & \\
\hline Repaired shunt lesion & 17 & 17 & 0 & \\
\hline Residual shunt lesion & 5 & 3 & 2 & \\
\hline Repaired valve lesion & 12 & 9 & 3 ( 2 deaths) & \\
\hline Unrepaired valve lesion & 3 & 3 & 0 & \\
\hline Other simple defect & 3 & 3 & 0 & \\
\hline Cardiac defect complexity, n (\%) & & & & 0.423 \\
\hline Complex & $25(24)$ & $20(22)$ & $5(38)$ & \\
\hline Moderately complex & $39(37)$ & $36(39)$ & $3(23)$ & \\
\hline Simple & $41(39)$ & $36(39)$ & $5(38)$ & \\
\hline Main defect related residual problems & & & & 0.089 \\
\hline No residual problems & $39(37)$ & $34(37)$ & $5(38)$ & \\
\hline Valvular problems & $38(36)$ & $36(39)$ & $2(15)$ & \\
\hline Heart failure & $10(10)$ & $9(10)$ & $1(8)$ & \\
\hline Arrhythmia & $11(10)$ & $9(10)$ & $2(15)$ & \\
\hline Pulmonary hypertension & $7(7)$ & $4(4)$ & $3(23)$ & \\
\hline
\end{tabular}

TGA includes patients with TGA after atrial switch, arterial switch and Rastelli-type procedure, as well as patients with congenitally corrected TGA; residual shunt lesion includes patients with a residual shunt after defect repair and patients with small, unrepaired shunts; other simple/moderately complex/complex defects include patients with corresponding lesions not included in any of the other categories.

${ }^{*} P$ value for the comparison of patients with and without complicated course.

ACHD, adult congenital heart disease; BMI, Body Mass Index; ES, Eisenmenger syndrome; TGA, transposition of the great arteries. 


\begin{tabular}{|c|c|c|c|}
\hline Variable & OR & $95 \% \mathrm{Cl}$ & $P$ value \\
\hline \multicolumn{4}{|l|}{ Univariable analysis } \\
\hline Male & 2.5 & 0.8 to 8.2 & 0.134 \\
\hline Age, per 5 years & 1.3 & 1.1 to 1.7 & 0.018 \\
\hline Comorbidities (two ore more) & 7.1 & 2.1 to 24.5 & 0.002 \\
\hline Overweight (BMl>25 kg/m²) & 7.2 & 1.9 to 28.3 & 0.004 \\
\hline Cyanotic heart disease/ES & 13.2 & 2.5 to 68.4 & 0.002 \\
\hline \multicolumn{4}{|l|}{ Multivariable analysis } \\
\hline Comorbidities (two or more) & 6.7 & 1.2 to 35.8 & 0.027 \\
\hline Overweight (BMl>25 kg/m²) & 16.4 & 3.2 to 83.4 & 0.001 \\
\hline Cyanotic heart disease/ES & 60.0 & 7.6 to 474.0 & $>0.001$ \\
\hline
\end{tabular}

Among the five patients who died during their hospital stay, COVID-19 related complications and the underlying cardiac defect were considered to interact and contribute to the fatal outcome in four cases. In one case (Patient 3 in table 4), coronavirus infection was considered likely not to be a major contributor to fatal outcome.

BMI, Body Mass Index; ES, Eisenmenger syndrome.

Data were analysed using STATA 15.1 statistical software. Distribution of continuous variables was assessed using visual inspection of the histogram and expressed as mean and SD for symmetrically distributed variables variables and as median and IQR for other data. Between-group comparisons in table 1 were performed using an unpaired Student's t-test or a $\chi^{2}$ test for continuous and nominal variables. Predictors of the main variable of interest (complicated clinical course, table 2) were analysed by univariable logistic regression. Due to the low number of events, multivariable analysis was restricted to variables with univariable OR of $>5$ (and a 95\% CI with the lower margin $>1$ ) and was calculated with cluster-robust SEs. Due to the sparsity of the outcome data, the ORs were recalculated in a second model with an exact logistic regression fit (see online supplemental material). In all analyses, the null hypothesis was rejected for $\mathrm{p}$ values of $<0.05$.

\section{Patient and public involvement}

There was no public or patient involvement in this study.

\section{RESULTS}

By 6 June, a total of 105 patients with ACHD were included in this study. Table 3 summarises the number of patients with ACHD per country reported to have COVID-19 and the number of yearly patient visits at the corresponding centres. Overall, 78 (74\%) patients had a confirmed diagnosis of COVID-19 by testing, while in 27 patients $(26 \%)$ the diagnosis was based on clinical grounds.

\begin{tabular}{lccl}
\hline Table 3 & Participating centres and reported cases \\
\hline Country & $\begin{array}{l}\text { ACHD centres } \\
\text { (n) }\end{array}$ & $\begin{array}{l}\text { COVID-19 cases (n } \\
\text { (\% of overall)) }\end{array}$ & $\begin{array}{l}\text { Yearly patients with } \\
\text { ACHD vits }\end{array}$ \\
\hline Spain & 6 & $50(48)$ & 12500 \\
Switzerland & 5 & $21(20)$ & 7000 \\
France & 1 & $13(12)$ & 2500 \\
Belgium & 4 & $12(11)$ & 6500 \\
The Netherlands & 3 & $5(5)$ & 5200 \\
Italy & 1 & $2(2)$ & 500 \\
Denmark & 3 & $1(1)$ & 8000 \\
Germany & 1 & $1(1)$ & 2500 \\
Austria & 1 & 0 & 1500 \\
Total & 25 & 105 & 46200 \\
\hline ACHD, adult congentital & &
\end{tabular}

ACHD, adult congenital heart disease.
At the time of data analysis, five patients (5\%) had died, and COVID-19 was still ongoing in nine cases (9\%). The infection had cleared in 2 patients (2\%) with sequelae, and 89 patients $(85 \%)$ recovered without additional new health problems. A total of 73 patients $(70 \%)$ had a mild course and did not require hospitalisation. Overall, a complicated disease course was observed in 13 cases (12\%), representing $41 \%$ (13/32) of the hospitalised patients. Table 1 summarises the characteristics of patients with ACHD in relation to the course of COVID-19. Details on patient characteristics and clinical history of patients with a complicated disease course are outlined in table 4 .

Patients with a complicated disease course were older and more likely overweight than patients with ACHD with uncomplicated COVID-19. In addition, these patients had more additional comorbidities. There was no significant gender difference in patients with and without a complicated COVID-19 course. Patients with two or more comorbidities were particularly at risk of complications. Overall cardiac defect complexity did not differ between patients with or without complicated COVID-19. A complicated disease course was more likely in patients with cyanotic heart disease, including Eisenmenger syndrome: four out of seven patients (57\%) had a complicated disease course. Among defect-related residual cardiac problems, pulmonary hypertension, present in seven patients (including five patients with Eisenmenger syndrome), had the strongest association with a complicated disease course.

In univariable analysis, age, overweight and multiple comorbidities were predictive of a complicated disease course (see table 2). When using a Body Mass Index (BMI) of $>25 \mathrm{~kg} / \mathrm{m}^{2}$ as cut-off, the corresponding OR was 7.2 (95\% CI 1.9 to 28.3, p=0.004). Among specific heart defects, highest risk for a complicated disease course was observed in unrepaired cyanotic heart defects or patients with Eisenmenger syndrome (figure 1). In a multivariable analysis of these three variables, all were independently associated with complications, and cyanotic heart disease was by far the most important predictor of a complicated disease course.

\section{DISCUSSION}

This is the first report on the outcome of COVID-19 in a sizeable cohort of European patients with ACHD with different types of congenital cardiac defects. The main findings of our study are the observation that risk factors derived from the general population, in particular, age, overweight and multiple comorbidities, are equally important for determining outcome in the ACHD population, in addition to the congenital cardiac defect. Congenital cardiac defects with very high risk for a complicated disease course in case of COVID-19 were unrepaired cyanotic heart disease or severe pulmonary hypertension with Eisenmenger syndrome. Such defects were present in 4 of 13 patients with a complicated course but represent only ca. $2 \%$ of all patients under follow-up at the participating centres. For other complex lesions, that is, univentricular physiology after Fontan palliation or defects with subaortic right ventricles, no such strong association was observed.

SARS-CoV-2 infection can cause both pulmonary and systemic inflammation, leading to acute respiratory distress syndrome and respiratory failure, sepsis, cardiac injury and thromboembolic complications, both in the venous and arterial circulations. ${ }^{9} 10$ Patients with cyanotic heart disease, including patients with Eisenmenger syndrome, exhibit chronic hypoxaemia with often markedly decreased resting oxygen saturations as a result of both a right-to-left shunt and severe abnormal pathobiology of the pulmonary tissue and pulmonary vascular bed. Such patients are at risk of rapid deterioration in case of respiratory tract infections with impaired oxygenation. In case of severe 


\begin{tabular}{|c|c|c|}
\hline Age (years) & Sex & Main diagnosis \\
\hline \multicolumn{3}{|c|}{ Fatal outcomes } \\
\hline $40-50$ & Male & Repaired tetralogy of Fallo \\
\hline$>60$ & Male & $\begin{array}{l}\text { Repaired pulmonary valve } \\
\text { stenosis }\end{array}$ \\
\hline $40-50$ & Female & $\begin{array}{l}\text { Bicuspid aortic valve with } \\
\text { severe aortic stenosis }\end{array}$ \\
\hline
\end{tabular}

50-60 Female Eisenmenger syndrome with unrepaired complete AVSD

40-50 Female Eisenmenger syndrome with unrepaired complete AVSD
Clinical background and disease course

Main cause of fatal outcome/ relation to congenital heart defect

Pre-existing severe biventricular dysfunction and progressive heart failure (had implanted (RT-D), cardiac-related liver cirrhosis and right lung hypoplasia due to an occluded right pulmonary artery; decision regarding cardiac and liver transplant was pending. Admitted with ARDS; due to comorbidities, the patient was not considered a candidate for extensive cardiorespiratory support; patient died at day 3 after hospital admission.

Mild pulmonary regurgitation, acquired cardiovascular disease (coronary artery disease, previous ischaemic stroke, abdominal aneurysm, atrial fibrillation) and COPD; NYHA class prior to COVID-19.

Admitted with bilateral pneumonia leading to ARDS requiring intubation on the day of admission; renal failure occurred 3 days after presentation; patient died on day 11 after admission with multiorgan failure.

Presentation with decompensated heart failure due to severe aortic stenosis, requiring urgent surgical aortic valve replacement; at admission, COVID-19 was not suspected; complicated postoperative course with cardiogenic shock requiring venoarterial ECMO.

Developed ARDS on first postoperative day and tested positive for SARS-CoV-2; patient died Comorbidities: one (heart failure) 7 days after surgery.

Severe pulmonary hypertension, heart failure and moderate leucopenia; presentation at the emergency department with bilateral pneumonia and ARDS.

Due to the patient's functional status (NYHA class III) prior to COVID-19 and personal preferences, she was transferred to a palliative care centre; she died on day 32 after initial hospital admission.

Severe pulmonary hypertension, heart failure and severely reduce renal function; presentation at the emergency department with ARDS.

Due to the patient's functional status (NYHA class IV) prior to COVID-19 and personal preferences, she was discharged home; patient died at home 22 days after initial hospital presentation.
Death due to SARS-CoV-2 infection (ARDS related to COVID-19)

Comorbidities: three (heart failure, liver and lung disease)

Death due to SARS-CoV-2 infection (ARDS related to COVID-19) coronary artery disease and lung disease)

Death with SARS-CoV-2 infection (postoperative death due to heart failure)

Death due to SARS-CoV-2 infection (ARDS related to COVID-19) Comorbidities: two (heart failure and pulmonary hypertension)

Death due to SARS-CoV-2 infection (ARDS related to COVID-19)

Comorbidities: three (heart failure pulmonary hypertension and kidney failure) Comorbidities: three (previous stroke
Patient with complicated disease course, ongoing cases 20-30 Male Unrepaired atrial secundum septal defect Down syndrome

50-60 Male Partial anomalous pulmonary venous connection, PFO with severe right-to-left shunt

\section{$30-40$ \\ Female Eisenmenger syndrome with persistent ductus arteriosus and atrial septal defect}

Recovered patients with complicated disease course

30-40 Female Fontan palliation for tricuspid atresia

30-40 Male Repaired ALCAPA

$>60 \quad$ Male Unrepaired CCTGA with VSD and residual severe pulmonary stenosis

Persistent right-to-left shunt through VSD with baseline oxygen saturation at $85 \%$

50-60 Male Repaired aortic coarctation Mechanical aortic valve replacement for severe aortic stenosis

50-60 Male Bentall procedure for bicuspid valvulopathy and aortopathy
History of bronchial asthma, NYHA class I prior to COVID-19.

Admitted with bilateral pneumonia and ARDS, requiring non-invasive ventilation; pulmonary thromboembolism occurred on day 4 after hospital admission; case still ongoing.

History of type 2 diabetes mellitus and oesophageal cancer; incidental diagnosis of partial anomalous partial anomalous pulmonary venous connection during the diagnostic cancer workup; normal right ventricular dimensions, no evidence of pulmonary hypertension. Hospital admission for elective oesophagectomy; at admission, COVID-19 not suspected; recurrent postoperative hypoxaemia requiring reintubation. Diagnosed with COVID-19 on postoperative day 4; subsequently severe ARDS with haemodynamic instability, severe pulmonary hypertension and multiple secondary infectious complications; diagnosis of a PFO with severe right-to-left shunting on postoperative day 23; emergent venoarterial ECMO on postoperative day 26 and percutaneous PFO closure on postoperative day 27 weaning from ECMO 7 days after PFO closure; case is still ongoing, slow recovery.

Obesity grade I (BMI $\left.33 \mathrm{~kg} / \mathrm{m}^{2}\right)$. NYHA class III prior to COVID-19.

ARDS secondary to bilateral pneumonia and bacterial superinfection; venoarterial ECMO

since day 1 of hospitalisation; a thromboembolic event occurred during hospitalisation; case Comorbidity: one (pulmonary still ongoing.

NYHA class II prior to COVID-19.

Admitted with bilateral pneumonia leading to ARDS, requiring intubation the day after of admission. The patient fully recovered 28 days after hospital admission.

NYHA class I prior to COVID-19

Admitted with cardiogenic shock requiring inotropic support for 4 days. Diagnosis of COVID19-related myocarditis; the patient fully recovered 27 days after hospital admission.

History of atrial flutter; NYHA class II prior to COVID-19

Admitted with bilateral pneumonia requiring transfer to the ICU for non-invasive ventilation at day 2 of hospitalisation; the patient experienced recurrent flutter during his hospitalisation and remained short of breath at last follow-up (NYHA III).

History of diabetes, stroke, heart failure with preserved ejection fraction and atria fibrillation; NYHA class II prior to COVID-19.

Admitted to the hospital with bilateral pneumonia requiring ICU transfer for non-invasive ventilation 2 days after admission; patient recovered 22 days after hospital admission, but impaired renal function persisted after hospital discharge.

History of diabetes and hypertension; NYHA class II previous to COVID-19.

Admitted with bilateral pneumonia requiring non-invasive ventilation; patient fully recovered 16 days after hospital admission.
Admission due to SARS-CoV-2 infection

Comorbidities: two (respiratory disease and genetic syndrome)

Admission for non-cardiac surgery PFO was a contributor to complicated disease course; partial anomalous pulmonary venous return likely not substantially contributing to disease course

Comorbidities: two (diabetes and cancer)

Admission due to SARS-CoV-2 infection

hypertension

Admission due to SARS-CoV-2

infection

Comorbidity: zero

Admission due to SARS-CoV-2

infection

Comorbidity: zero

Admission due to SARS-CoV-2

infection

Comorbidity: zero

Admission due to SARS-CoV-2 infection

Comorbidities: three (diabetes, stroke and heart failure)

Admission due to SARS-CoV-2

infection

Comorbidities: two (diabetes and arterial hypertension)

ALCAPA, This footnote is not necessary - no such abbreviation is used in table 4

;ARDS, acute respiratory distress syndrome; AVSD, atrioventricular septal defect; BMI, Body Mass Index; CCTGA, congenitally corrected transposition of the great arteries; COPD, chronic obstructive pulmonary disease; CRT-D, cardiac resynchronisation therapy defibrillator; ECMO, extracorporeal membrane oxygenation; ICU, intensive care unit; NYHA, New York Heart Association; ; PFO, persistent foramen ovale; VSD, ventricular septal defect. 


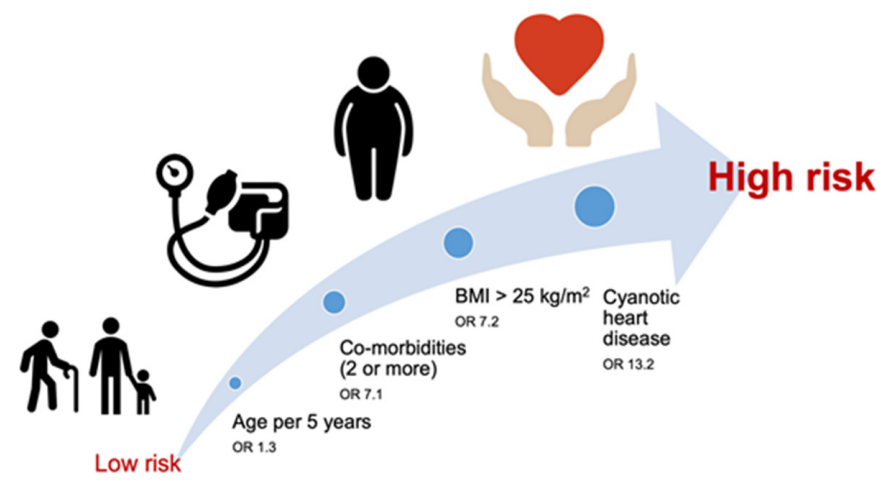

Figure 1 Univariable significant COVID-19 risk factors in patients with adult congenital heart disease and the corresponding ORs. We propose to stratify patients based on age, number of comorbidities, weight and presence of a high-risk cardiac lesion (cyanotic heart disease). BMI, Body Mass Index.

COVID-19, pre-existing hypoxaemia can be exacerbated by an increase in right-to-left shunting due to the rise in pulmonary vascular resistance and an inflammation-related decrease in systemic vascular resistance. ${ }^{11}$ These patients are furthermore at increased risk of paradoxical embolism. The potentially increased prothrombotic risk due to pre-existing haemostatic abnormalities, venous stasis, endothelial injury and inflammatory response may also contribute to a worse outcome in these patients. ${ }^{9}$

Our study underscores the importance of a comprehensive risk assessment, not only taking into account the underlying congenital defect but also, more importantly, considering general risk factors and comorbidities for risk estimation in case of COVID-19. The most important general risk factor for COVID-19-related mortality is age. ${ }^{12}$ In line with previous studies, we observed an increasing risk of complications with advancing age. At present, the median age of patients with ACHD followed up at specialised centres is 35 years, and the prevalence of adults with CHD older than 60 years is estimated at $5 \%-10 \%$ of the entire ACHD population only. ${ }^{14} 15$ This young age may make them less susceptible for virus invasion, and hence patients with ACHD tend to have a milder COVID-19 course. ${ }^{16}$ This may explain why patients with univentricular physiology after Fontan palliation-mostly young adults-were at lower risk of a complicated disease course as intuitively anticipated by the anatomical absence of a subpulmonary ventricle.

Overweight emerged as a risk factor in our study, independent of other comorbidities and defect complexity. This was not the case in a large cohort study from New York, with a median patient age of 62 years, and $46 \%$ of them being obese, ${ }^{17}$ nor in the first reports from China ${ }^{12}$ or Italy. ${ }^{18}$ In line with our findings, Kass et al described an inverse correlation between age and BMI among patients admitted with COVID-19 to the Johns Hopkins Hospital, in which younger individuals with severe disease were more likely obese. ${ }^{19}$ Our study supports their hypothesis that, among younger patients with less comorbidities, obesity may play a more important role than among elderly patients with multiple comorbidities for predicting COVID-19 outcomes.

Acquired cardiovascular and other comorbidities associated with fatal COVID-19 outcomes are infrequently found in the ACHD population. In the Spanish ACHD cohort, 75\% of patients were younger than 45 years, and the prevalence
Key messages

What is already known on this subject?

- The novel SARS-CoV-2 responsible for COVID-19 is thought to interact with the cardiovascular system on multiple levels, leading to increased morbidity and mortality in patients with underlying cardiovascular diseases.

What might this study add?

- Whether patients with adult congenital heart disease (ACHD) should be considered to be at increased risk of poor outcomes if suffering from COVID-19 is unclear. This is the first observational study providing clinical evidence in this respect.

How might this impact on clinical practice?

- So far, COVID-19 risk stratification in patients with ACHD was based on expert opinion. Our cohort study provides observational evidence regarding COVID-19 risk factors in patients with ACHD and improves tailoring of recommendations for preventive measures in individual patients.

of hypertension, diabetes and ischaemic heart disease was only $14 \%, 2.7 \%$ and $1.5 \%$, respectively. Still, even in young patients with ACHD with fewer comorbidities than encountered in an elderly population, the presence of multiple comorbidities confers a markedly increased risk of a complicated disease course. The observed OR of two or more comorbidities for a complicated disease course in this study was 7.1 (95\% CI 2.1 to 24.5 ) in the univariable regression model and 6.7 (95\% CI 1.2 to 35.8 ) in the multivariable regression model. Hence, patients with ACHD with multiple comorbidities should be considered as vulnerable patients, independent of the underlying defect complexity.

There are limitations inherent to this study. First, we did not systematically test all patients with ACHD under follow-up at participating centres for COVID-19. Hence, we may have missed cases, with most likely mild disease course. As a consequence, we are not able to provide data regarding the prevalence and disease course of COVID-19 among the ACHD population. Currently, the paper describes outcome data of only $0.2 \%$ of the ACHD population followed by the centres in the past year, a number certainly lower than the prevalence of COVID-19 among the general population. Second, despite data collection in 25 European ACHD centres with more than 46000 yearly patient visits, the absolute number of COVID-19 cases among patients with ACHD was still small, limiting statistical analysis. The small sample size may also explain the non-significant $\mathrm{p}$ value for gender differences related to a complicated COVID-19 course, despite different proportions observed between the groups. Due to the lack of observational data at the beginning of the pandemic, many patients with ACHD were routinely advised to adhere to the concept of physical distancing and personal protection with strict hygiene measures. These recommendations, backed up by a self-perception of being at risk, may have effectively prevented COVID-19 cases and contributed to the overall low number of infected patients with ACHD. The small sample size resulted in large CIs. Further confirmation of our results by other study groups is necessary. In this respect, the results of another cohort study supported by 
the International Society for Adult Congenital Heart Disease are eagerly awaited.

In conclusion, our study provides first evidence that, among patients with ACHD advanced age, obesity and multiple comorbidities are associated with an increased risk of a complicated COVID-19 course, independent of the underlying cardiac defect. Congenital cardiac defects at particular high risk of a complicated disease course in case of COVID-19 were cyanotic lesions, including unrepaired cyanotic defects or severe pulmonary hypertension with Eisenmenger syndrome. These data can be used to identify patients with ACHD more vulnerable for a complicated COVID-19 course. Given the paucity of data so far, further confirmation of our findings is needed.

\section{Author affiliations}

Center for Congenital Heart Disease, Inselspital University Hospital, Bern, Switzerland

${ }^{2}$ Dept. of Cardiology III - Adult Congenital and Valvular Heart Disease, University Hospital Muenster, Munster, Nordrhein-Westfalen, Germany

${ }^{3}$ Department of Cardiology, Academic Medical Center, Amsterdam, The Netherlands ${ }^{4}$ Department of Cardiology and Cardiac Surgery, Lausanne University Hospital, Lausanne, Switzerland

${ }^{5}$ Division of Cardiology, University Hospital of Geneva, Geneva, Switzerland ${ }^{6}$ Congenital and Structural Cardiology, University Hospital Leuven, Leuven, Flanders, Belgium

${ }^{7}$ Department of Cardiovascular Sciences, Catholic University Leuven, Leuven, Flanders, Belgium

${ }^{8}$ Department of Cardiology, Ghent University Hospital, Gent, Oost-Vlaanderen, Belgium

${ }^{9}$ ACHD UNIT - Pediatric and Adult Congenital Heart Centre, IRCCS - Policlinico San Donato, San Donato Milanese - Milan, Lombardia, Italy

${ }^{10}$ Pediatric Cardiology and GUCH Centre, Ramon y Cajal University Hospital, Madrid, Spain

${ }^{11}$ Department of Cardiology, Medical University of Vienna, Vienna, Austria

${ }^{12}$ Adult Congenital Heart Disease Unit, Department of Cardiology, Hospital Universitario, Virgen del Rocio, Instituto de BioMedicina de Sevilla (IBIS) and CIBERCV, Sevilla, Spain

${ }^{13}$ Adult Congenital Heart Disease Unit, Department of Cardiology, Hospital Universitario Virgen de las Nieves. 2 Instituto de Investigación Biosanitaria ibs, Granada, Andalucía, Spain

${ }^{14}$ Hospital Universitario La Paz, Madrid, Spain

${ }^{15}$ Department of Cardiology, Rigshospitalet, Copenhagen University Hospital, Copenhagen, Denmark

${ }^{16}$ Adult Congenital Heart Disease Unit, Centre de référence des Malformations Cardiaques Congénitales Complexes, M3C, Université de Paris, Hôpital Européen Georges Pompidou, AP-H, Paris, France

${ }^{17}$ Integrated Adult Congenital Heart Disease Unit, Vall d'Hebron University Hospital and Santa Creu i Sant Pau University Hospital, Barcelona, Spain

${ }^{18}$ Department of Cardiology, CHU Brugmann, Brussels, Belgium

${ }^{19}$ Department of Cardiology, Cliniques Universitaires Saint-Luc, Bruxelles, Belgium

${ }^{20}$ Adult Congenital Heart Disease Unit, Department of Cardiology, Hospital Universitari i Politècnic La Fe and CIBERCV, Valencia, Comunidad Valenciana, Spain

${ }^{21}$ Department of Cardiology, Erasmus Medical Centre, Rotterdam, The Netherlands

${ }^{22}$ Department of Cardiology, University Medical Centre Utrecht, Utrecht, The

Netherlands

${ }^{23}$ Department of Cardiology, University Hospital Basel, University of Basel, Basel,

Switzerland

${ }^{24}$ Departament of Cardiology, University Heart Center, University Hospital Zurich, Zurich, Switzerland

Twitter Markus Schwerzmann @MarkusSchwerzm1 and Pastora Gallego @ apgalgar

Collaborators European Collaboration for Prospective Outcome Research in Congenital Heart Disease: Anissa Boubrit, Université de Paris, Hôpital Européen Georges Pompidou, Paris, France; Francisco Buendía Fuentes, Adult Congenital Heart Disease Unit, Department of Cardiology, Hospital Universitari i Politècnic La Fe and CIBERCV, València, Spain; Julie De Backer, Department of Cardiology, Ghent University Hospital, Ghent, Belgium; Michèle De Hosson, specialist nurse, Department of Cardiology, Ghent University Hospital, Ghent, Belgium; Laura Dos Subirà. Unitat Integrada de Cardiopaties Congènites de I'Adolescent i de l'Adult Vall d'Hebron-Sant Pau, Department of Cardiology, Vall d'Hebron University Hospital and CIBERCV, Barcelona, Spain; Eduardo Moreno Escobar, Adult Congenital Heart Disease Unit, Department of Cardiology. Hospital Universitario Virgen de las Nieves. Granada. Spain. 2 Instituto de Investigación Biosanitaria ibs. GRANADA, Granada, Spain; Dorte Guldbrand Nielsen, Department of Cardiology, Skejby, Aarhus University Hospital, Denmark; Sophie Pierrad, Pôle de Recherche Cardiovasculaire, Institut de Recherche Expérimentale et Clinique, Université Catholique de Louvain and Divisions of Cardiology and Cardiothoracic Surgery, Cliniques Universitaires Saint-Luc, Brussels, Belgium; Maria-Jose Rodriguez-Puras, Adult Congenital Heart Disease Unit, Department of Cardiology, Hospital Universitario Virgen del Rocio. Sevilla, Spain.

Contributors MS, FJR-R, DT, MG and JB contributed in the drafting of the manuscript, conception of the research, critical revision of the manuscript for important intellectual content and supervision. All other authors contributed in the patient recruitment and data collection, critical revision of the manuscript for important intellectual content and supervision.

Funding The European Collaboration for Prospective Outcome Research in Congenital Heart Disease is funded by internal grants without support from the pharmaceutical industry.

Competing interests WB declared being proctor of Abbott and Occlutech.

Patient consent for publication Not required.

Ethics approval The study complied with the Declaration of Helsinki and was approved by local research ethics committees according to local ethical policies and country-specific regulations.

Provenance and peer review Not commissioned; externally peer reviewed.

Data availability statement Data are available upon reasonable request.

Supplemental material This content has been supplied by the author(s). It has not been vetted by BMJ Publishing Group Limited (BMJ) and may not have been peer-reviewed. Any opinions or recommendations discussed are solely those of the author(s) and are not endorsed by BMJ. BMJ disclaims all liability and responsibility arising from any reliance placed on the content. Where the content includes any translated material, BMJ does not warrant the accuracy and reliability of the translations (including but not limited to local regulations, clinical guidelines, terminology, drug names and drug dosages), and is not responsible for any error and/or omissions arising from translation and adaptation or otherwise.

Open access This is an open access article distributed in accordance with the Creative Commons Attribution Non Commercial (CC BY-NC 4.0) license, which permits others to distribute, remix, adapt, build upon this work non-commercially, and license their derivative works on different terms, provided the original work is properly cited, appropriate credit is given, any changes made indicated, and the use is non-commercial. See: http://creativecommons.org/licenses/by-nc/4.0/.

\section{ORCID iDs}

Markus Schwerzmann http://orcid.org/0000-0002-4006-8929

Francisco Javier Ruperti-Repilado http://orcid.org/0000-0002-9904-0402

Laurence Campens http://orcid.org/0000-0002-5045-2449

Massimo Chessa http://orcid.org/0000-0001-7432-4815

Pastora Gallego http://orcid.org/0000-0003-2115-5047

Annette Schophuus Jensen http://orcid.org/0000-0003-4170-6416

Annemien $\mathrm{E}$ van den Bosch http://orcid.org/0000-0002-0422-9860

Daniel Tobler http://orcid.org/0000-0002-0821-3196

\section{REFERENCES}

1 European Centre for Disease Prevention and Control. COVID-19 situation update for the EU/EEA and the UK. Available: https://www.ecdc.europa.eu/en/2019-ncovbackground-disease

2 Baumgartner $\mathrm{H}$, Budts $\mathrm{W}$, Chessa $\mathrm{M}$, et al. Recommendations for organization of care for adults with congenital heart disease and for training in the subspecialty of 'Grown-up Congenital Heart Disease' in Europe: a position paper of the Working Group on Grown-up Congenital Heart Disease of the European Society of Cardiology. Eur Heart J 2014;35:686-90.

3 Diller G-P, Kempny A, Alonso-Gonzalez R, et al. Survival prospects and circumstances of death in contemporary adult congenital heart disease patients under follow-up at a large tertiary centre. Circulation 2015;132:2118-25.

4 Oliver JM, Gallego P, Gonzalez AE, et al. Risk factors for excess mortality in adults with congenital heart diseases. Eur Heart J 2017;38:1233-41.

5 Raissadati A, Nieminen H, Haukka J, et al. Late causes of death after pediatric cardiac surgery: a 60-year population-based study. J Am Coll Cardiol 2016;68:487-98.

6 Lui GK, Saidi A, Bhatt AB, et al. Diagnosis and management of noncardiac complications in adults with congenital heart disease: a scientific statement from the American heart association. Circulation 2017;136:e348-92.

7 Diller G-P, Gatzoulis MA, Broberg CS. Coronavirus disease 2019 in adults with congenital heart disease: a position paper from the ESC Working group of adult 
congenital heart disease, and the International Society for adult congenital heart disease. Eur Heart J 2020;323.

8 Baumgartner H, De Backer J, Babu-Narayan SV, et al. 2020 ESC guidelines for the management of adult congenital heart disease. Eur Heart J

2021:42:563-645.

9 Bikdeli B, Madhavan MV, Jimenez D, et al. COVID-19 and thrombotic or thromboembolic disease: implications for prevention, antithrombotic therapy, and follow-up: JACC state-of-the-art review. J Am Coll Cardiol 2020:75:2950-73

10 Guzik TJ, Mohiddin SA, Dimarco A, et al. COVID-19 and the cardiovascular system: implications for risk assessment, diagnosis, and treatment options. Cardiovasc Res 2020;116:1666-87.

11 Creel-Bulos C, Hockstein M, Amin N, et al. Acute cor pulmonale in critically ill patients with Covid-19. N Eng/ J Med 2020;382:e70.

12 Zhou F, Yu T, Du R, et al. Clinical course and risk factors for mortality of adult inpatients with COVID-19 in Wuhan, China: a retrospective cohort study. Lancet 2020;395:1054-62.
13 Du R-H, Liang L-R, Yang C-Q, et al. Predictors of mortality for patients with COVID-19 pneumonia caused by SARS-CoV-2: a prospective cohort study. Eur Respir J 2020;55:2000524.

14 Tutarel 0, Kempny A, Alonso-Gonzalez R, et al. Congenital heart disease beyond the age of 60: emergence of a new population with high resource utilization, high morbidity, and high mortality. Eur Heart J 2014;35:725-32.

15 Baumgartner $\mathrm{H}$. Geriatric congenital heart disease: a new challenge in the care of adults with congenital heart disease? Eur Heart J 2014;35:683-5.

16 Nikolich-Zugich J, Knox KS, Rios CT, et al. SARS-CoV-2 and COVID-19 in older adults: what we may expect regarding pathogenesis, immune responses, and outcomes. Geroscience 2020:42:505-14.

17 Cummings MJ, Baldwin MR, Abrams D, et al. Epidemiology, clinical course, and outcomes of critically ill adults with COVID-19 in New York City: a prospective cohort study. The Lancet 2020;395:1763-70.

18 Onder G, Rezza G, Brusaferro S. Case-Fatality rate and characteristics of patients dying in relation to COVID-19 in Italy. JAMA 2020;323:1775-6.

19 Kass DA, Duggal P, Cingolani O. Obesity could shift severe COVID-19 disease to younger ages. Lancet 2020;395:1544-5. 